\title{
Non-convexity of level sets in convex rings for semilinear elliptic problems
}

\author{
R. Monneau \\ Ecole Nationale des Ponts et Chaussées, CERMICS, 6 et 8 avenue Blaise Pascal, \\ Cité Descartes Champs-sur-Marne, 77455 Marne-la-Vallée Cedex 2, France \\ H. Shahgholian \\ Department of Mathematics, Royal Institute of Technology, \\ 10 044, Stockholm, Sweden, \\ E-mail: henriksh@math.kth.se
}

November 16, 2003

\begin{abstract}
We show that there is a convex ring $R=\Omega^{-} \backslash \Omega^{+} \subset \mathbf{R}^{2}$ in which there exists a solution $u$ to a semilinear partial differential equation

$$
\Delta u=f(u), \quad u=-1 \text { on } \partial \Omega^{-}, \quad u=1 \text { on } \partial \Omega^{+},
$$

with level sets, not all convex. Moreover every bounded solution $u$ has at least one nonconvex level set. In our construction, the nonlinearity $f$, is non-positive, and smooth.
\end{abstract}

AMS Classification: 35J60, 35R35.

Keywords: Non-convexity, level set, semilinear elliptic equation, convex ring.

\section{Introduction}

Consider, in $\mathbf{R}^{2}$, two nested convex domains $\Omega^{-} \supset \Omega^{+}$and the convex ring $R=\Omega^{-} \backslash \overline{\Omega^{+}}$. In [14] it was proved that under conditions

$$
f \in L^{1}(-\infty,+\infty), \quad f \geq 0, \quad f=0 \quad \text { on } \quad(-\infty,-1)
$$


there exists a weak solution $u$ to the following boundary value problem

$$
\left\{\begin{array}{l}
\Delta u=f(u) \quad \text { on } \quad R=\Omega^{-} \backslash \bar{\Omega}^{+} \\
u=-1 \quad \text { on } \partial \Omega^{-} \\
u=1 \text { on } \partial \Omega^{+},
\end{array}\right.
$$

having convex level sets $\{u \geq \lambda\}$. The second author and M. Poghosyan are trying to extend this result in higher dimensions, and to the case of $p$-Laplacian [16].

Here we prove the following counter-example.

Theorem 1.1 There exist a convex ring $R \subset \mathbf{R}^{2}$, a smooth function $f$ satisfying

$$
f \leq 0 \quad \text { on }(-\infty,+\infty), \quad f=0 \quad \text { on } \quad(-\infty,-1) \cup(0,+\infty),
$$

and a solution to (1.1), with a non-convex level set. Moreover every bounded solution to (1.1) has at least one non-convex level set.

Let us remark that there exists some related literature on the breaking of radial symmetry in annuli, see for instance the well-known article of Brezis and Nirenberg [4], and [10].

\section{$2 \quad$ Proof of theorem 1.1}

The proof of theorem 1.1 is based on the one hand on the following counter-exemple of Acker [1] (see also [2]) on a free boundary problem, see also [14].

Theorem 2.1 There exists a convex ring $R=\Omega^{-} \backslash \overline{\Omega^{+}} \subset \mathbf{R}^{2}$ and a constant $q>0$ such that for every smooth domain $\Omega$ such that

$$
\Omega^{+} \subset \subset \Omega \subset \subset \Omega^{-},
$$

and every bounded solution $u$ to the following problem:

$$
\left\{\begin{array}{l}
\Delta u=0 \quad \text { on } \quad R \backslash \partial \Omega \\
u=-1 \quad \text { on } \quad \partial \Omega^{-} \\
u=+1 \quad \text { on } \quad \partial \Omega^{+} \\
u=0 \quad \text { on } \quad \partial \Omega \\
\left(\frac{\partial u^{+}}{\partial n}\right)^{2}-\left(\frac{\partial u^{-}}{\partial n}\right)^{2}=-q^{2}<0 \quad \text { on } \quad \partial \Omega
\end{array}\right.
$$


the domain $\Omega$ is not convex.

On the other hand we will use the following result on the approximation of the free boundary solution by a solution to a semilinear elliptic problem (which is a consequence of a more general result for parabolic equations [9]).

Theorem 2.2 (Caffarelli, Lederman, Wolanski [9])

Let $\beta$ be a nonnegative, Lipschitz continuous function, supported in $[-1,0]$, such that

$$
\|\beta\|_{L^{\infty}(\mathbf{R})} \leq M_{0}<+\infty \quad \text { and } \quad \int_{\mathbf{R}} \beta(s) d s=\frac{q^{2}}{2}
$$

and let us define

$$
\beta_{\varepsilon}(s)=\frac{1}{\varepsilon} \beta\left(\frac{s}{\varepsilon}\right) .
$$

We consider a sequence of bounded solutions $u^{\varepsilon}$ to the following problem

$$
\left\{\begin{array}{l}
\Delta u^{\varepsilon}=-\beta_{\varepsilon}\left(u^{\varepsilon}\right) \quad \text { on } \quad R=\Omega^{-} \backslash \overline{\Omega^{+}} \\
u^{\varepsilon}=-1 \quad \text { on } \quad \partial \Omega^{-} \\
u^{\varepsilon}=+1 \quad \text { on } \quad \partial \Omega^{+} .
\end{array}\right.
$$

Let us assume that $u^{\varepsilon} \longrightarrow u$ uniformly on an open set $D \subset \subset R$. If for some point $X_{0} \in \partial\{u<0\} \cap D$, the set $\{u<0\}$ has an inward unit normal $\nu$ in the following sense

$$
\lim _{r \rightarrow 0^{+}} \frac{1}{r^{2}} \int_{B_{r}\left(X_{0}\right) \cap\{u<0\}}\left|1_{\{u<0\}}-1_{\left\{<X-X_{0}, \nu><0\right\}}\right|=0
$$

and if the set $\{u=0\}$ has a zero Lebesgue density at $X_{0}$, i.e.

$$
\lim _{r \rightarrow 0^{+}} \frac{1}{r^{2}} \int_{B_{r}\left(X_{0}\right)} 1_{\{u=0\}}=0
$$

then we have

$$
u(X)=\alpha<X-X_{0}, \nu>^{+}-\beta<X-X_{0}, \nu>^{-}+o\left(\left|X-X_{0}\right|\right)
$$

for some $\alpha, \beta \geq 0$ verifying

$$
\alpha^{2}-\beta^{2}=-q^{2}<0 .
$$

Proof of theorem 1.1

Step 1: Construction of a solution $u^{\varepsilon}$ of (2.2)

Let $\underline{u}$ be the solution to

$$
\left\{\begin{array}{lll}
\Delta \underline{u}=0 & \text { on } & R \\
\underline{u}=+1 & \text { on } & \partial \Omega^{+} \\
\underline{u}=-1 & \text { on } & \partial \Omega^{-}
\end{array}\right.
$$


By construction $\underline{u}$ is a subsolution to problem (2.2).

Next, for $\delta>0$, consider the one-dimensional solution $w^{\varepsilon}(r)$

$$
\left\{\begin{array}{l}
w_{r r}^{\varepsilon}+\frac{1}{r} w_{r}^{\varepsilon}=-\beta_{\varepsilon}\left(w^{\varepsilon}\right) \quad \text { on } \quad(\delta, 2 \delta) \\
w^{\varepsilon}(\delta)=-1 \\
w^{\varepsilon}(2 \delta)=+1
\end{array}\right.
$$

where $w_{r}^{\varepsilon}$ is the first derivative of $w^{\varepsilon}$ with respect to $r$, and $w_{r r}^{\varepsilon}$ is the second derivative of $w^{\varepsilon}$. As $\varepsilon \longrightarrow 0$, we have $w^{\varepsilon} \longrightarrow w^{0}$ uniformly on $(\delta, 2 \delta)$, where for some $c \in(\delta, 2 \delta)$ and $\alpha, \beta \geq 0$, we have

$$
w^{0}(r)=\left\{\begin{array}{lll}
-1+c \alpha \ln \left(\frac{r}{\delta}\right) & \text { if } & r \in[\delta, c] \\
+1+c \beta \ln \left(\frac{r}{2 \delta}\right) & \text { if } \quad r \in[c, 2 \delta]
\end{array}\right.
$$

such that $\alpha^{2}-\beta^{2}=-q^{2}<0$ and $w^{0}(c)=0$. It is then easy to see that there exists a unique such solution $(c, \alpha, \beta)$. Let

$$
W^{\varepsilon}(X)=\left\{\begin{array}{lll}
w^{\varepsilon}(|X|) & \text { if } & \delta<|X|<2 \delta \\
-1 & \text { if } & |X| \leq \delta \\
+1 & \text { if } & |X| \geq 2 \delta
\end{array}\right.
$$

which is a supersolution on $B_{2 \delta} \backslash \bar{B}_{\delta}$, i.e. satisfies $\Delta W^{\varepsilon} \leq-\beta_{\varepsilon}\left(W^{\varepsilon}\right)$ on $B_{2 \delta} \backslash \bar{B}_{\delta}$. Let

$$
\bar{u}^{\varepsilon}(X)=\inf _{B_{\delta}\left(X_{0}\right) \subset \mathbf{R}^{2} \backslash \Omega^{-}} W^{\varepsilon}\left(X-X_{0}\right) .
$$

Then $\bar{u}^{\varepsilon}$ is a supersolution which, for $\delta$ small enough (independently on $\varepsilon$ ), satisfies

$$
\bar{u}^{\varepsilon}=1 \quad \text { on } \quad \Omega^{+} .
$$

Now from Perron's method, we deduce the existence of a solution $u^{\varepsilon}$ of $(2.2)$ which satisfies

$$
\underline{u} \leq u^{\varepsilon} \leq \bar{u}^{\varepsilon} \quad \text { on } R .
$$

Let us remark that every bounded solution $u^{\varepsilon}$ to (2.2) satisfies (2.3). In particular this proves that there exists some $\eta>0$ independent on $\varepsilon$ such that

$$
\left\{\begin{array}{l}
\left\{u^{\varepsilon} \geq \eta\right\} \supset\left\{X, \quad \operatorname{dist}\left(X, \Omega^{+}\right) \leq \eta\right\} \\
\left\{u^{\varepsilon} \leq-\eta\right\} \supset\left\{X, \quad \operatorname{dist}\left(X, \mathbf{R}^{2} \backslash \Omega^{-}\right) \leq \eta\right\} .
\end{array}\right.
$$




\section{Step 2: Passage to the limit $\varepsilon \longrightarrow 0$}

We want to prove that for $f=-\beta_{\varepsilon}$ and for $\varepsilon$ small enough, every solution $u^{\varepsilon}$ of (1.1) has at least one non-convex levet set $\left\{u^{\varepsilon}>\lambda\right\}$. Suppose this fails. Then for all $\lambda \in(-1,1)$, and some subsequence $\varepsilon_{n} \rightarrow 0$, the level sets $\left\{u^{\varepsilon_{n}}>\lambda\right\}$ are convex.

We define

$$
R_{\eta}=\{X \in R, \quad \operatorname{dist}(X, \partial R)>\eta\},
$$

and recall that from (2.4) and Proposition 2.1 in [8] we have the following result.

Lemma 2.3 There exists a constant $L_{\eta}>0$ such that

$$
\left|\nabla u^{\varepsilon_{n}}\right| \leq L_{\eta} \quad \text { on } \quad R_{2 \eta}
$$

In particular, we can pass to the limit as $\varepsilon_{n}$ goes to zero and we get the existence of a limit function $u$ defined on $R$ such that

$$
\begin{aligned}
& u^{\varepsilon_{n}} \longrightarrow u \quad \text { uniformly on } R_{2 \eta}, \\
& \left\{\begin{array}{lll}
\Delta u=0 & \text { on } & R \backslash\{=0\} \\
\Delta u \leq 0 & \text { on } & R \\
u=1 & \text { on } & \partial \Omega^{+} \\
u=-1 & \text { on } & \partial \Omega^{-}
\end{array}\right.
\end{aligned}
$$

and

$$
\left\{\begin{array}{l}
\{u \geq \eta\} \supset\left\{X, \quad \operatorname{dist}\left(X, \Omega^{+}\right) \leq \eta\right\} \\
\{u \leq-\eta\} \supset\left\{X, \quad \operatorname{dist}\left(X, \mathbf{R}^{2} \backslash \Omega^{-}\right) \leq \eta\right\} \\
|\nabla u| \leq L_{\eta} \quad \text { on } \quad R_{2 \eta}
\end{array} .\right.
$$

Moreover, because of our assumptions, we have

$$
\{u>\lambda\} \quad \text { are convex for all } \lambda \in(-1,1) .
$$

The latter, in particular, will be shown to imply

Proposition 2.4 The assumptions of theorem 2.2 are fulfilled.

Therefore Theorem 2.2 implies that the limit $u$ (of $u^{\varepsilon_{n}}$ ) is a viscosity solution of problem (2.1), in the sense of Caffarelli [5]. Then the theory of Caffarelli $[6,7]$ proves that the free boundary $\{u=0\}$ is a $C^{1, \alpha}$ curve. From the results of Kinderlehrer, Nirenberg [12], we deduce that the free boundary is analytic. Therefore $u$ satisfies (2.1) and 
$\Omega=\{u>0\}$ is convex because of (2.5). This contradicts Theorem 2.1. This ends the proof of Theorem 1.1.

\section{Proof of proposition $\mathbf{2 . 4}$}

To prove proposition 2.4, we only have to prove that assumptions of Theorem 2.2 are fulfilled, which is a consequence of the following two lemmata:

Lemma 2.5 We have $\{u=0\}^{0}=\emptyset$.

Lemma 2.6 $\partial \Omega$ is $C^{1}$ (no corners).

\section{Proof of lemma 2.5}

If $\{u=0\}^{0} \neq \emptyset$, then, in virtue of the convexity of level sets, we can find a ball in $\{u>0\}$, tangent to $\{u=0\}$ at a point $X_{0}$. Then from Hopf lemma $\frac{\partial u^{+}}{\partial \nu} \neq 0$. As a consequence we get for the limit $u^{0}$ of $u^{\lambda}=\frac{u\left(X_{0}+\lambda X\right)}{\lambda}$ as $\lambda$ goes to zero:

$$
u^{0}(X)=\alpha<X, \nu>^{+} \quad \text { for } \quad \alpha=\left|\frac{\partial u^{+}}{\partial \nu}\right|>0 .
$$

In particular

$$
\Delta u^{0} \geq 0 \quad \text { and } \quad \Delta u^{0} \not \equiv 0 .
$$

This is in contradiction with the fact that $\Delta u^{\lambda} \leq 0$ for $\lambda>0$.

\section{Proof of lemma 2.6}

Assume that $\{u>0\}$ has a corner at $X_{0}=0$, i.e.

$$
\{u>0\} \subset\{X=(r \cos \theta, r \sin \theta), \quad r>0, \quad \theta \in(-\pi, \pi], \quad|\theta|>\beta\},
$$

for some $\beta>\pi / 2$. For $\gamma=\frac{\pi}{2 \beta}<1$, we consider the function

$$
v^{\beta}(X)=\left\{\begin{array}{l}
r^{\gamma} \cos (\gamma \theta) \quad \text { if } \quad|\theta| \leq \beta \\
0 \text { if } \quad|\theta|>\beta
\end{array} .\right.
$$

Then $-A v^{\beta}$ is a supersolution on $R \backslash \bar{\Omega}$ for $A>0$ small enough, and it is above $u$ on $\left\{v^{\beta}>0\right\}$. But $u$ is Lipschitz inside $R_{2 \eta}$ and thus a contradiction.

\section{Acknowledgements}

First author thanks Göran Gustafsson foundation for a visiting appointment to the Royal Institute of Technology. Second author was supported in part by Swedish Research Council. The authors also would like to thank an unknown referee for constructive suggestions. 


\section{References}

[1] A. ACKER, On the Non-Convexity of Solutions in Free-Boundary problems Arising in Plasma Physics and Fluid Dynamics, Comm. Pure Appl. Math. 42, 1165-1174, (1989).

[2] A. ACKER, Addendum to: "On the nonconvexity of solutions in free-boundary problems arising in plasma physics and fluid dynamics", Comm. Pure Appl. Math. 42 (1989), no. 8, 1165-1174

[3] Berestycki H., Caffarelli, L.A., Nirenberg L., Uniform Estimates for Regularization of Free Boundary Problems, Analysis and partial differential equations, Coll. Pap. dedic. Mischa Cotlar, Lect. Notes Pure Appl. Math. 122, 567-619 (1990).

[4] H. Brezis And L. Nirenberg, Positive solutions of nonlinear elliptic equations involving critical Sobolev exponents., Comm. Pure. Appl. Math., 41, 437-477, (1983).

[5] Caffarelli, L.A., A Harnack Inequality Approach to the Regularity of Free Boundaries, Part I: Lipschitz Free Boundaries are $C^{1, \alpha}$, Rev. Mat. Iberoamericana 3 (2), 139-162, (1987).

[6] Caffarelli, L.A., A Harnack Inequality Approach to the Regularity of Free Boundaries, Part II: Flat Free Boundaries are Lipschitz, Comm. Pure Appl. Math. 42, 55-78, (1989).

[7] Caffarelli, L.A., A Harnack Inequality Approach to the Regularity of Free Boundaries, Part III: Existence Theory, Compactness, and Dependence on X, Ann. Scuola Norm. Sup. Pisa Cl. Sci 15 (4), 583-602, (1989).

[8] L.A. Caffarelli, C. Lederman, N. Wolanski, Uniform Estimates and Limits for a Two Phase Parabolic Singular Perturbation Problem, Indiana Univ. Math. J. 46, No.2, 453-489 (1997).

[9] L.A. Caffarelli, C. Lederman, N. Wolanski, Pointwise and Viscosity Solutions for a Two Phase Parabolic Singular Perturbation Problem, Indiana Univ. Math. J. 46, No.3, 719-740 (1997).

[10] Catrina Florin, Zhi-Qiang Wang, Nonlinear elliptic equations on expanding symmetric domains., J. Differ. Equations 156, No.1, 153-181, (1999).

[11] D. DANIELLI, A singular perturbation approach to a two phase parabolic free boundary problem arising in combustion theory, preprint. 
[12] Kinderlehrer, D., Nirenberg, L., Regularity in free boundary problems, Ann. Scuola Norm. Sup. Pisa Cl. Sci 4, 373-391, (1977).

[13] Laurence P., Stredulinsky E., Existence of Regular Solutions with Convex Levels for Semilinear Elliptic Equations with Nonmonotone $L^{1}$ Nonlinearities, Indiana Univ. Math. J., 39 (4), 1081-1114, (1990).

[14] Laurence P., Stredulinsky E., Existence of Regular Solutions with Convex Levels for Semilinear Elliptic Equations with Non-monotone $L^{1}$ Nonlinearities, Part II. Passage to the limit, Indiana Univ. Math. J., , 485-498.

[15] C. Lederman, J.L. Vasquez, N. Wolanski, Uniqueness of solution to a free boundary problem from combustion, to appear in Trans. Amer. Math. Soc..

[16] M. Poghosyan, H. Shahgholian, in preparation. 